\title{
A Numerical Survey of the Floors of Various Hilbert Fundamental Domains
}

\section{By Harvey Cohn}

1. Introduction. From the purely computational point of view we are considering a real positive function $S_{2}=f\left(R_{1}, R_{2}, S_{1}\right)$ defined (in (3.15) below) for values $R_{1}, R_{2}$, and $S_{1}$ varying on a parallelopiped. The function $f$ is composed of a large and undetermined number (possibly thousands!) of analytic pieces. The object is to find the minimum of $f$ and to estimate the number of pieces which constitute $f$. What we do is probably the easiest thing: We subdivide the parallelopiped by a regular three-dimensional grid and scan for $\min S_{2}$ as well as the number of pieces in the function $f$.

The function $f$ arises here in an interesting context, however, since it represents part of the boundary (called the "floor") of the fundamental domain $\boldsymbol{R}$ for Hilbert's modular group for certain quadratic fields of unique factorization. (We assume some knowledge of factorization theory [1] but we summarize Siegel's theory of these fundamental domains [2], [5], for easy reference.) It is important to know the minimum of $S_{2}$ (and the optimal "low-point") because of applications to relative-quadratic fields $[2, \S 6],[4]$ and it is important to know the number of pieces constituting $f$ as a clue to the topological structure of $\boldsymbol{R}$ under boundary identifications.

The most important single result is the Main Theorem of $\$ 5$ (below). It states that among quadratic fields of unique factorization, with the only exception of the field of $5^{1 / 2}$, no fundamental domain of the corresponding Hilbert modular group can have a "simple floor" or a floor consisting of "a single piece" (if we ignore translates; these terms will be explained more precisely below). The proof is based on counterexamples which were yielded by a computational search process.

The computer here serves as a crude instrument since it may fail to discern all pieces of the surface being examined or it may overestimate the "low-point" on the surface. Still we must not discount the possibility that a thorough round-off analysis could establish an error estimate for the scanning, which could be used, provided vast amounts of computing time became available.

Grateful acknowledgment is made for the use of the CDC 3600 at the Argonne National Laboratories of the USAEC and for the cooperation of Dr. William F. Miller, director of the Applied Mathematics Division, and Mr. Burton S. Garbow, programmer.

2. Summary of Theory. We consider the quadratic field $K$ generated by $k^{1 / 2}$ where $k$ is a square-free integer $>1$ and where the field in question has classnumber unity. We take 12 cases:

$$
k=2,3,5,6,7,11,13,14,17, \cdots, 21,29,33, \cdots .
$$

We let Greek letters denote integers in $\boldsymbol{K}$ and "primed" Greek letters denote con-

Received October 26, 1964. Supported by NSF grant G-7412. 
jugates, while lower-case Roman letters denote rational integers. We let $\boldsymbol{O}$ denote the ring of integers in $\boldsymbol{K}$; thus $\boldsymbol{O}$ is the set of

$$
\alpha=x+y \omega, \quad \omega=\left[(c-1)+k^{1 / 2}\right] / c
$$

and $c=2$ if $k \equiv 1(\bmod 4)$ while $c=1$ otherwise. We call $\alpha$ totally positive $(\alpha \gg 0)$ when $\alpha>0$ and $\alpha^{\prime}>0$, and we let $\epsilon_{+}(>1)$ denote the fundamental totally positive unit. (The identification of $\epsilon_{+}$with the fundamental unit $\epsilon_{0}$ or with $\epsilon_{0}^{2}$ is shown in Table 1 below.)

Consider now the bicomplex upper half planes $U \times U$

$$
Z=X+i Y, \quad Z^{\prime}=X+i Y^{\prime} \quad\left(Y>0, Y^{\prime}>0\right),
$$

where continuous variables are denoted by capital Roman letters. The primes here denote formal relations so that norm $N$ and trace $S$ can apply equally well to the complex variables as to the algebraic numbers. Thus $N(\alpha Y)=\alpha \alpha^{\prime} Y Y^{\prime}$, $\boldsymbol{S}(\alpha Y)=\alpha Y+\alpha^{\prime} Y^{\prime}$, etc. We call

$$
\|Z\|=N\left(X^{2}+Y^{2}\right)=\left(X^{2}+Y^{2}\right)\left(X^{\prime 2}+Y^{\prime 2}\right)
$$

and we call the height

$$
h\left(Z, Z^{\prime}\right)=N(Y)=Y Y^{\prime} .
$$

The Hilbert modular group $H$ is the group of transformations of $U \times U$ onto itself

$$
Z \rightarrow(\alpha Z+\beta) /(\gamma Z+\delta), \quad Z^{\prime} \rightarrow\left(\alpha^{\prime} Z^{\prime}+\beta^{\prime}\right) /\left(\gamma^{\prime} Z^{\prime}+\delta^{\prime}\right)
$$

where the determinant is a totally positive unit or

$$
\alpha \delta-\beta \gamma=\epsilon \gg 0 .
$$

The indicated transformations divide the space $U \times U$ into equivalence classes and a fundamental domain $R$ for $H$ is a minimal subset of $U \times U$ representing each equivalence class (exactly once). The choice of $R$ is not definite but, in principle, one desires to have a conveniently small number of bounding (three dimensional) manifolds. The exact number is of such manifolds still unknown; all we show is that this number becomes quite large as $k$ increases, according to the computations.

An important subgroup of $H$ is $H_{\infty}$ which leaves fixed the point at $\infty \quad(Z=\infty$, $\left.Z^{\prime}=\infty\right)$. It consists of the transformations

$$
Z \rightarrow \epsilon Z+\beta, \quad Z^{\prime} \rightarrow \epsilon^{\prime} Z+\beta^{\prime}
$$

where $\epsilon \gg 0$ is a unit. A fundamental domain for $H_{\infty}$ is rather elementary (see $[2, \S 4])$; it is seen to be given by $\boldsymbol{R}_{\infty}$ which is the cartesian product of a wedge and parallelogram:

$$
\begin{gathered}
\epsilon_{+}{ }^{-1} \leqq Y^{\prime} / Y \leqq \epsilon_{+}, \\
-\frac{1}{2} \leqq R_{1} \leqq \frac{1}{2}, \quad-\frac{1}{2} \leqq R_{2} \leqq \frac{1}{2},
\end{gathered}
$$

where we use the computationally convenient variables $R_{1}, R_{2}$ defined by

$$
X=R_{1}+\omega R_{2}, \quad X^{\prime}=R_{1}+\omega^{\prime} R_{2} .
$$

Of course boundary points are identified through (2.7) in obvious fashion. 
It also can be shown that of all points in each equivalence class created by (2.5) there exist (several) points where $\boldsymbol{h}\left(Z, Z^{\prime}\right)$ is maximized. Such points are characterized by the fact they belong in the domain given by (see $[2, \S 4])$,

$$
R_{0}=\bigcap_{(\gamma, \delta)}\{Z:\|\gamma Z+\delta\| \geqq 1\},
$$

where $\gamma, \delta$ range over $O$ but $\gamma \neq 0$. A fundamental domain for $H$ can be shown to be the intersection

$$
R=R_{0} \cap R_{\infty}
$$

(with boundary points identified according to $H$ this time). It can be further shown that for $\left(Z, Z^{\prime}\right) \in R_{0}$,

$$
\min h\left(Z, Z^{\prime}\right)=h_{0}(k)>c^{2} /(2 k),
$$

and it is actually achieved at some low point $\left(Z_{0}, Z_{0}^{\prime}\right)$ of $R$ (see $[2, \S 4]$ ). The points of $R$ which lie on some hypersurface $\|\gamma Z+\delta\|=1$ are said to constitute the floor of $R$. If for all points of the floor we can associate a $(\gamma, \delta)$ with $\gamma=1$, we say that the floor is simple, in effect this means the floor consists essentially of $\|Z\|=$ 1 (and its "translates" $Z \rightarrow Z+\delta$ ).

For a point on the floor $Z$, we have $\|\gamma Z+\delta\|=1$ for some $(\gamma, \delta)$ but $\|\gamma Z+\delta\|>1$ for all others (i.e., we never have $\|\gamma Z+\delta\|<1$ ). In many cases the decimal accuracy in distinguishing the inequality will be very critical.

3. Description of Program. The floor of the fundamental domain is a threedimensional set in four-dimensional space. Therefore, if we fix any point $\left(X, X^{\prime}\right)$ or $\left(R_{1}, R_{2}\right)$ in the parallelogram $(2.9 \mathrm{ab})$ the floor would determine a curve $C$ in $\left(Y, Y^{\prime}\right)$ space.

To parametrize $C$ it is more convenient to introduce new coordinates $S_{1}, S_{2}$ analogous with $R_{1}, R_{2}$ as follows:

$$
S_{1}=k^{1 / 2}\left(Y^{\prime}-Y\right) /\left(Y^{\prime}+Y\right)
$$

and, because of our interest in the height $\boldsymbol{h}\left(Z, Z^{\prime}\right)$,

$$
S_{2}=Y Y^{\prime} \text {. }
$$

The advantage in using $S_{1}$ consists in the fact that the wedge (2.8) becomes transformed into the parallel strip:

$$
-h \leqq S_{1} \leqq h
$$

where (recalling $\epsilon_{+}{ }^{\prime}=\epsilon_{+}{ }^{-1}$ ) we see the bounds are rational:

$$
h=k^{1 / 2}\left(\epsilon_{+}-1\right) /\left(\epsilon_{+}+1\right)(>0) .
$$

Values of $h$ are shown in Table 1 (see below; $\Delta S_{1}$ is presently explained in (3.14)).

The curve $C$ in the floor lying over each $\left(X, X^{\prime}\right)$ or $\left(R_{1}, R_{2}\right)$ can be parametrized by the ratio $Y^{\prime} / Y$ or by $S_{1}$ in the range (3.3). To see this, define

$$
\Phi_{0}\left(Z, Z^{\prime}\right)=\inf _{(\gamma, \delta)}\|\gamma Z+\delta\|, \quad(0 \neq \gamma \in O, \delta \in O) .
$$


TABLE 1

Range of $S_{1}$

\begin{tabular}{r|l|l|l|l}
\hline$k$ & \multicolumn{1}{c|}{$\epsilon_{0}$} & $\epsilon_{+}$ & $h$ & \multicolumn{1}{c}{$\Delta S_{1}$} \\
\hline 2 & $1+2^{1 / 2}$ & $\epsilon_{0}^{2}$ & 1 & .2 \\
3 & $2+3^{1 / 2}$ & $\epsilon_{0}$ & 1 & .2 \\
6 & $5+2 \cdot 6^{1 / 2}$ & $\epsilon_{0}$ & 2 & .1 \\
7 & $8+3 \cdot 7^{1 / 2}$ & $\epsilon_{0}$ & 3 & $.333 \cdots$ \\
11 & $10+3 \cdot 11^{1 / 2}$ & $\epsilon_{0}$ & 3 & .3 \\
14 & $15+4 \cdot 14^{1 / 2}$ & $\epsilon_{0}$ & $\frac{7}{2}$ & .35 \\
5 & $\frac{1}{2}\left(1+5^{1 / 2}\right)$ & $\epsilon_{0}{ }^{2}$ & 1 & .2 \\
13 & $\frac{1}{2}\left(3+13^{1 / 2}\right)$ & $\epsilon_{0}^{2}$ & 3 & .3 \\
17 & $4+17^{1 / 2}$ & $\epsilon_{0}^{2}$ & 4 & .5 \\
21 & $\frac{1}{2}\left(5+21^{1 / 2}\right)$ & $\epsilon_{0}$ & 3 & .3 \\
29 & $\frac{1}{2}\left(5+29^{1 / 2}\right)$ & $\epsilon_{0}{ }^{2}$ & 5 & .5 \\
33 & $23+4 \cdot 33^{1 / 2}$ & $\epsilon_{0}$ & $\frac{11}{2}$ & .55 \\
\hline
\end{tabular}

Clearly $\Phi_{0}$ increases monotonically in $G$ where $Z=X+G Y i, Z^{\prime}=X^{\prime}+G Y^{\prime} i$. Thus for each fixed $S_{1}$ there is precisely one $S_{2}$ for which $\Phi_{0}=1$.

Actually these functions are computationally quite reasonable. We note the inequalities:

$$
\|\gamma Z+\delta\| \geqq N(\gamma)^{2} N(Y)^{2}
$$

and

$$
\begin{aligned}
& \|\gamma Z+\delta\|>\left(\gamma^{\prime} Y^{\prime}\right)^{2}(\gamma X+\delta)^{2}, \\
& \|\gamma Z+\delta\|>(\gamma Y)^{2}\left(\gamma^{\prime} X^{\prime}+\delta^{\prime}\right)^{2} .
\end{aligned}
$$

Hence $\|\gamma Z+\delta\|>1$ unless (by (2.12))

$$
0<|N(\gamma)|<2 k / c^{2}
$$

and

$$
\begin{aligned}
& -\gamma X-\left|\gamma^{\prime} Y^{\prime}\right|^{-1}<\delta<-\gamma X+\left|\gamma^{\prime} Y^{\prime}\right|^{-1}, \\
& -\gamma^{\prime} X^{\prime}-|\gamma Y|^{-1}<\delta^{\prime}<-\gamma^{\prime} X^{\prime}+|\gamma Y|^{-1} .
\end{aligned}
$$

Only a finite set of "input" $\gamma$ satisfies (3.8) (see Table 3 below) if we ignore associates of any $\gamma$ (values of $\gamma$ differing by a unit factor). For each $\gamma$ a finite set of $\delta$ and $\delta^{\prime}$ are determined by (3.9) (see Remark (a) in $\$ 8$ below). The set of admissible $\gamma$ was worked out beforehand by hand, using factorization laws for the field $\boldsymbol{K}$. These values are stored in the memory as couples $\left(g_{1}, g_{2}\right)$ where $\gamma=$ $g_{1}+g_{2} \omega$. For any given $Z$ it is no difficult task to program a computer to run through all $\gamma$ and $\delta$ satisfying (3.8) and (3.9). Call this set $\boldsymbol{M}$. Then for practical purposes we are dealing, not with $\Phi_{0}$ of (3.5) above but with the numerically equal function $\Phi_{1}$ (written in $R_{1}, R_{2}, S_{1}, S_{2}$ instead of $Z, Z^{\prime}$ for convenience),

$$
\Phi_{1}\left(R_{1}, R_{2}, S_{1}, S_{2}\right)=\min \|\gamma Z+\delta\|, \quad((\gamma, \delta) \in M) .
$$

Incidentally the fact that $\boldsymbol{M}$ is a finite set makes the "inf" of (3.5) an actual 
minimum. (It also assures us that infinitely many different boundary surfaces " $\|\gamma Z+\delta\|=1$ " will not accumulate near a point $Z, Z^{\prime}$ as long as $Y>0$, $Y^{\prime}>0$.) The machine does keep track, additionally, of the last $(\gamma, \delta)$ which produces the minimum in (3.10), for later purposes of output.

We can finally calculate the curve $C$ lying over $\left(R_{1}, R_{2}\right)$ (or $\left(X, X^{\prime}\right)$ ) by solving for $S_{2}$ so that the floor is now given by one "finitary" equation:

$$
\Phi_{1}\left(R_{1}, R_{2}, S_{1}, S_{2}\right)=1 .
$$

By virtue of the earlier remarks (see (2.12)) there is a unique solution

$$
2 /\left(c^{2} k\right)<S_{2} \leqq 1
$$

and this value of $S_{2}$ can be found by a bisection process on $S_{2}$ in the interval (3.12). designed to cut off when $\left|\Phi_{1}-1\right|<E$ a preassigned value (see Remark (b) of $\$ 8$ below). As a check on accuracy, the machine prints out the difference "error $S_{2}$ " between the last two approximations to $S_{2}$ (by bisection). In practice with $E=.001$, usually "error $S_{2}$ " $<.01$.

Now consider the inverse of $(3.11)$ as $S_{2}=f\left(R_{1}, R_{2}, S_{1}\right)$. For this function, it is easily seen that if $k \not \equiv 1(\bmod 4)$ there is complete " $\pm R_{1}$ and $\pm R_{2}$ symmetry." This follows from the fact that we can change $X$ and $X^{\prime}$ to $-X$ and $-X^{\prime}$ while we can interchange $X$ and $Y$ with $X^{\prime}$ and $Y^{\prime}$. (If $k \equiv 1(\bmod 4)$ the symmetry is not reflected in $R_{1}$ and $R_{2}$ both, e.g., only "symmetry about the origin" can be used.) Thus we search the following parallelopiped:

$$
\begin{gathered}
-.5 \leqq R_{1} \leqq 0 \text { in } N_{1} \text { steps of } \Delta R_{1}\left(\Delta R_{1}=.1, N_{1}=6\right), \\
-.5 \leqq R_{2} \leqq 0 \text { in } N_{2} \text { steps of } \Delta R_{2}\left(\Delta R_{2}=.1, N_{2}=6\right), \\
-h \leqq S_{1} \leqq h \text { in } N_{3} \text { steps of } \Delta S_{1} \quad \text { (Table 1). }
\end{gathered}
$$

(We use $-.5 \leqq R_{2} \leqq .5$ if $k \equiv 1 \bmod 4$ since we lack one of the symmetries.) Here $\Delta R_{1}, \Delta R_{2}, \Delta S_{1}$ are part of the input data as well as the numbers $N_{1}, N_{2}$, $N_{3}$ of steps (and of course the starting values $-.5,-.5$ and $-h$ ).

The output represents essentially the floor as determined by inverting (3.11) to obtain

$$
S_{2}=f\left(R_{1}, R_{2}, S_{1}\right) ;
$$

it consists of the sequence of $N_{1} N_{2} N_{3}$ "points" in 13 columns:

$$
R_{1}, R_{2}, S_{1}, S_{2}, \quad X, X^{\prime} \quad Y, Y^{\prime}, \quad g_{1}, g_{2}, d_{1}, d_{2},
$$

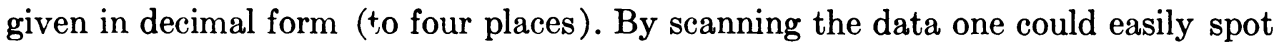
low points as small values of $S_{2}$. The values of $\gamma=g_{1}+\omega g_{2}$ and $\delta=d_{1}+\omega d_{2}$ are printed to correspond to the last value of $(\gamma, \delta) \in \boldsymbol{M}$ arising (in finding $S_{2}$ ) for which $\Phi_{1}$ takes its minimum. This way, if we are dealing with a nonsimple floor we should find $\gamma \neq 1$ or $\left(g_{1}, g_{2}\right) \neq(1,0)$. More will be said about the output of $(\gamma, \delta)$ later on (see Table 3 below).

4. Study of Case $k=6$. To illustrate the main run for a typical case take $k=6$. Here the function of (3.14), $S_{2}=f\left(R_{1}, R_{2}, S_{1}\right)$ is calculated for the following $1476=6 \cdot 6 \cdot 41$ points 


$$
\begin{array}{lll}
-.5 \leqq R_{1} \leqq 0, & \Delta R_{1}=.1 & (6 \text { values }) \\
-.5 \leqq R_{2} \leqq 0, & \Delta R_{2}=.1 & (6 \text { values }) \\
-2 \leqq S_{1} \leqq 2, & \Delta S_{1}=.1 & (41 \text { values }) .
\end{array}
$$

Let us concentrate on each curve $C$ which lies over $\left(R_{1}, R_{2}\right)$. It is represented by 41 points $\left(S_{1}, S_{2}\right)$ which in turn lie on a (variable) number of hypersurfaces " $\|\gamma Z+\delta\|=1$ ". For example, when $R_{1}=-.3$ and $R_{2}=-.4$, the print-outs reveal that $\boldsymbol{C}$ consists of five analytic pieces lying on the following hypersurfaces:

\begin{tabular}{|c|c|c|c|c|c|c|c|c|c|c|c|}
\hline-.5 & & -.4 & & -.3 & & -.2 & & -.1 & & 0 & $/$ \\
\hline${ }_{*}^{A}$ & & ${ }_{*}^{A}$ & & ${ }_{*}^{A}$ & & ${ }_{*}^{A}$ & & A & & A & 0 \\
\hline .33 & & .47 & & .64 & & .81 & & .95 & & 1.00 & \\
\hline & $\stackrel{A}{*}$ & & $A_{*}$ & & $\stackrel{A}{*}$ & & $\underset{*}{A}$ & & ${ }_{*}^{A}$ & & \\
\hline & .40 & & .38 & & .52 & & .70 & & .86 & & \\
\hline$A$ & & $A_{*}^{A}$ & & ${ }_{*}^{A}$ & & ${ }_{*}^{A}$ & & ${ }_{*}^{A}$ & & ${ }_{*}^{A}$ & -.1 \\
\hline .69 & & .50 & & .33 & & .41 & & .57 & & .74 & \\
\hline & $\underset{*}{A}$ & & $A$ & & $A_{*}$ & & ${ }_{*}^{A}$ & & ${ }_{*}^{*}$ & & \\
\hline & .68 & & .61 & & .41 & & .41 & & .45 & & \\
\hline${ }_{*}^{A}$ & & ${ }_{*}^{A}$ & & ${ }_{*}^{A}$ & & ${ }_{*}^{A}$ & & ${ }_{*}^{A}$ & & A & -.2 \\
\hline .62 & & .63 & & .61 & & .51 & & .31 & & .41 & \\
\hline & ${ }_{*}^{A}$ & & ${ }_{*}^{A}$ & & ${ }_{*}^{A}$ & & $A_{*}$ & & ${ }_{*}^{A}$ & & \\
\hline & .56 & & .55 & & .49 & & .45 & & .35 & & \\
\hline${ }_{*}^{A}$ & & ${ }_{*}^{A}$ & & ${ }_{*}^{A}$ & & ${ }_{*}^{A}$ & & ${ }_{*}^{A}$ & & ${ }_{*}^{A}$ & -.3 \\
\hline .45 & & .46 & & .45 & & .36 & & .28 & & .40 & \\
\hline & $\stackrel{A}{*}$ & & $\underset{*}{A}$ & & $A_{*} B$ & & $A \underset{*}{A B C}$ & & $A_{*} B$ & & \\
\hline & .34 & & .33 & & .27 & & .31 & & .34 & & \\
\hline$A_{*} B$ & & ${ }_{*} B$ & & $A_{*}^{A B C}$ & & $A_{*} B$ & & $A_{*} B$ & & $A_{*} B$ & -.4 \\
\hline .25 & & .20 & & .26 & & .32 & & .39 & & .43 & \\
\hline & $A \underset{*}{A B D}$ & & ${ }_{*}^{A B C}$ & & ${ }_{*}^{A B}$ & & $A_{*} B$ & & $A_{*} B$ & & \\
\hline & .24 & $A B$ & .26 & $A B$ & .36 & $A B$ & .42 & $A B$ & .46 & $A B$ & -5 \\
\hline * & & $*$ & & $*$ & & $*$ & & * & & $*$ & \\
\hline .25 & & .29 & & .37 & & .44 & & .48 & & .50 & \\
\hline
\end{tabular}

$$
\begin{aligned}
& \|Z-1\|=1 \text { for }-2.0 \leqq S_{1} \leqq-1.7 \text {, } \\
& \left\|\left(3+6^{1 / 2}\right) Z+\left(2+6^{1 / 2}\right)\right\|=1 \text { for }-1.6 \leqq S_{1} \leqq-1.0 \text {, } \\
& \left\|\left(2+6^{1 / 2}\right) Z+\left(3+6^{1 / 2}\right)\right\|=1 \text { for }-.9 \leqq S_{1} \leqq 1.0 \text {, } \\
& \|Z+1\|=1 \text { for } \quad 1.1 \leqq S_{1} \leqq 1.9 \text {, } \\
& \left\|Z-1+6^{1 / 2}\right\|=1 \text { for } 2.0=S_{1} \text {. }
\end{aligned}
$$

The values of $S_{2}$ vary, generally displaying several relative minima for the range

Fig. 1.-Value of $\min S_{2}$ as $S_{1}$ varies for fixed values of $R_{1}$ and $R_{2}$ (between -.5 and 0 ) on the lower left-hand quadrant. 
of $S_{1}$; indeed $S_{2}$ increases from .260 at $S_{1}=-2$ to .529 at $S_{1}=1.7$ finally decreasing to .499 at $S_{1}=2$ (ignoring minor fluctuations). In Figure 1 we indicate the minimum (computed) value of $S_{2}$ over the range $-2 \leqq S_{1} \leqq 2$ as a function of $R_{1}$ and $R_{2}$ alone, i.e., we indicate the minimum value of $S_{2}$ on $C$ for each of 36 $\left(R_{1}, R_{2}\right)$. In order to give a better picture of how this minimum varies, we also calculate the values at the 25 mid-points of the squares by a supplementary computation.

Moreover, to obtain some idea of how complicated the "piecing" of hypersurfaces becomes, in Figure 1, we denote by

$$
\begin{aligned}
& A \text { the presence of }|N(\gamma)|=1, \\
& B \text { the presence of }|N(\gamma)|=2, \\
& C \text { the presence of }|N(\gamma)|=3, \\
& D \text { the presence of }|N(\gamma)|=4
\end{aligned}
$$

among the hypersurfaces $\|\gamma Z+\delta\|=1$ occurring over $\left(R_{1}, R_{2}\right)$. Thus the last point, $R_{1}=-.3, R_{2}=-.4$, bears the information " $A B C, .26$ ", etc. We see that " $D$ " occurs only twice, in the lower left-hand corner. Clearly, we can fail to find hypersurfaces by round-off error as well as by using a grid which is not sufficiently fine, so that we must regard results of this nature as tentative and unreliable indications of a minimum degree of complication. (Of course, several different $\delta$ can occur with each $\gamma$ but they are not distinguished here.)

Finally, the low-point suggested by Figure 1 is

$$
R_{1}=-.4, \quad R_{2}=-.4, \quad S_{1}=-1, \quad S_{2}=.2
$$

or, in the original coordinate,

$$
\begin{aligned}
& Z=-\frac{2}{5}\left(1+6^{1 / 2}\right)+\frac{i}{5}\left(1+6^{1 / 2}\right), \\
& Z^{\prime}=-\frac{2}{5}\left(1-6^{1 / 2}\right)+\frac{i}{5}\left(-1+6^{1 / 2}\right) .
\end{aligned}
$$

Subsequent exploration with a finer grid seems to justify the conjecture that (4.1) or (4.2) is the low-point (see Remark (c) in $\$ 8$ below).

5. Nonsimplicity Theorem. We now examine the output data to see where $\gamma \neq 1$. This is a necessary condition for a nonsimple floor (but not a sufficient one since we can have the coincidental occurrence $\|\gamma Z+\delta\|=\left\|Z+\delta_{1}\right\|=1$, i.e., the boundary point $Z$ can lie on two boundary surfaces at once).

Our attention is immediately drawn to the special values $R_{1}=0, R_{2}=-1 / 2$, for which the (floor) output shows $\gamma \neq 1$ (except when $k=3$ or 5 ). The values of $S_{1}$ and $S_{2}$ are not always the same but for simplicity we consider the point $Z_{*}$ corresponding to $S_{1}=0$ and $S_{2}=1 / 4$ or

$$
Z_{*}=\frac{1}{2}(-\omega+i), \quad Z^{\prime}{ }_{*}=\frac{1}{2}\left(-\omega^{\prime}+i\right) .
$$

Now $Z_{*}$ does not generally lie on the floor, but still 


$$
\left\|2 Z_{*}+\omega\right\|=1 \text {. }
$$

We shall soon try to see if for all $\delta \in O$ the following holds for $Z=Z_{*}$ :

$$
\|Z+\delta\|>1 \text {. }
$$

From (5.2) it follows that in $(3.11) \Phi_{1}(0,-1 / 2,0,1 / 4) \leqq 1$. Thus if $S_{2}{ }^{\prime}$ is the root of $\Phi_{1}\left(0,-1 / 2,0, S_{2}{ }^{\prime}\right)=1$, it follows that $S_{2}{ }^{\prime} \geqq 1 / 4$ and (5.3) still holds for the point of the floor corresponding to this root. Hence, (see $[2, \S 8]$ ) there is no hypersurface of type (5.3) on the floor at $R_{1}=0, R_{2}=-1 / 2, S_{1}=0, S_{2}{ }^{\prime}(\geqq 1 / 4)$.

Actually to demonstrate (5.3) for $Z=Z_{*}$, consider its negation, $16 \| Z_{*}+$ $\delta \| \leqq 16$. This can be expanded as

$$
\left(\sigma^{2}+1\right)\left(\sigma^{\prime 2}+1\right) \leqq 16
$$

where $\sigma=2 \delta-\omega$, which is irrational when $\delta$ varies in $O$. Clearly (5.4) provides a bound on $|\sigma|$ and $\left|\sigma^{\prime}\right|$ hence only a finite number of quadratic fields can permit the inequality (5.4). A quick check reveals the only counterexamples satisfying (5.4) and $\sigma \equiv \omega(\bmod 2)$ are

$$
\begin{aligned}
\sigma= \pm\left(\frac{1+5^{1 / 2}}{2}\right), \pm\left(\frac{-3+5^{1 / 2}}{2}\right) & \\
& \pm 2^{1 / 2}, \pm 3^{1 / 2}, \pm 2 \pm 3^{1 / 2}, \pm\left(\frac{-3+13^{1 / 2}}{2}\right) .
\end{aligned}
$$

Therefore, the only cases to check are $k=5,2,3,13$. Actually $k=5$ is easily disposed of as simple from (3.8) since in $K,|N(\gamma)|=1$ or $\geqq 4$. To dispose of $k=2,3$ and $k=13$ we search the outputs again and we obtain the following counterexamples by trial and error:

$k=2$. Let

$$
Z_{*}=-\frac{1}{2} \cdot 2^{1 / 2}+i, \quad Z_{*}{ }^{\prime}=\frac{1}{2} \cdot 2^{1 / 2}+\frac{i}{2} .
$$

Thus $\left\|2^{1 / 2} Z_{*}+1\right\|=1$ while if $\delta=m+n 2^{1 / 2}$, then

$$
\left\|Z_{*}+\delta\right\|=\left\{\left(m+\left[n-\frac{1}{2}\right] 2^{1 / 2}\right)^{2}+1\right\}\left\{\left(m-\left[n-\frac{1}{2}\right] 2^{1 / 2}\right)^{2}+\frac{1}{4}\right\} .
$$

But $\left\|Z_{*}+\delta\right\| \leqq 1$ only if $\left|m^{2}-2\left(n-\frac{1}{2}\right)^{2}\right|<1$ or if $(2 n-1)+m 2^{1 / 2}$ is a unit. Then trying units, we find $\left\|Z_{*}+\delta\right\| \geqq 9 / 8>1$ for $\delta \in O$.

$k=3$. Let $Z_{*}=\left(-4-5 \cdot 3^{1 / 2}+7 i\right) / 10, Z_{*}{ }^{\prime}=\left(-4+5 \cdot 3^{1 / 2}+7 i\right) / 10$ (not an easy point to conjecture but it appeared on the print-outs with $\gamma=1+3^{1 / 2}$, $\left.\delta=2+3^{1 / 2}\right)$ ! Here

$$
\left\|\left(1+3^{1 / 2}\right) Z_{*}+\left(2+3^{1 / 2}\right)\right\|=\left\|\left(1+3^{1 / 2}\right)(1+7 i) / 10\right\|=1 .
$$

Meanwhile, after somewhat more labored calculation we verify

$$
\left\|Z_{*}+\delta\right\| \geqq \frac{37}{25}>1 \text { for } \delta \in O \text {. }
$$

$k=13$. This case resembles $k=2$ (see Remark (d) of $\S 8$ below). Let

$$
Z_{*}=-\frac{1}{2} \omega+i, \quad Z_{*}{ }^{\prime}=-\frac{1}{2} \omega^{\prime}+\frac{i}{4}, \quad\left(\omega=\frac{1}{2}\left[1+13^{1 / 2}\right]\right) .
$$


Thus $\left\|2 Z_{*}+\omega\right\|=1$ while with $\delta=m+n \omega$ we note that $\|Z+\delta\|>1$ unless, with $\lambda=2 m+(2 n-1) \omega,|N(\lambda)| \leqq 4$. But if $N(\lambda)= \pm 1, \pm 3$, since $\|Z+\delta\|>(\lambda / 8)^{2}+\left(\lambda^{\prime} / 2\right)^{2}$ we reduce our choice (by $\left.\max |\lambda|,\left|\lambda^{\prime}\right|<8\right)$ to $\lambda=$ $\pm\left(3-13^{1 / 2}\right) / 2, \pm\left(1+13^{1 / 2}\right) / 2, \pm\left(5+13^{1 / 2}\right) / 2 ;($ recall $\lambda \equiv \omega \bmod 2)$. By a close margin, again,

$$
\left\|Z_{*}+\delta\right\| \geqq(806-150 \sqrt{13}) / 256=1.03 \cdots>1(!) .
$$

MaIN Theorem. The only quadratic field of class number unity with a simple floor for the Hilbert fundamental domain is the field of $5^{1 / 2}$.

In completing this proof, we did not depend on the print-outs, but we verified all inequalities rigorously. In what follows we shall depend on the print-outs because of the amount of labor involved in verifying even a single (print-out) $S_{2}$.

6. Low-Points. We first inspect the print-outs in order to conjecture the minimum value of $S_{2}$. (A more difficult job, of course, is to invent some justification for the conjectured minimum.) In certain cases, $k=2,3,5,6,13$ a reasonable conjecture could be made from this and earlier work ([2], [3]). In other cases, $k=11,21,29,33$ no "exact" conjecture seems available so we content ourselves with repeating the decimal values in the output. In still other cases, however, $k=7,14,17$, we can "explain" the minimum output but without sufficient conviction to justify a conjecture that this value is the low-point.

A frequent situation for some $\boldsymbol{K}$ is where the output produces $S_{1}=0$, or $Y=$ $Y^{\prime}\left(=Y_{k}\right)$. Then the precise value of $Y_{k}$ is determined by the condition

$$
\|\gamma Z+\delta\|=\left[(\gamma X+\delta)^{2}+\gamma^{2} Y_{k}^{2}\right]\left[\left(\gamma^{\prime} X^{\prime}+\delta^{\prime}\right)^{2}+\gamma^{\prime 2} Y_{k}{ }^{2}\right]=1
$$

drawn from the output values of $\gamma$ and $\delta$. Then $S_{2}=Y_{k}^{2}$. This happens for $k=$ $2,3,7,13,14,17$.

The relevant information is collected in final form in Table 2 . In each case, the

TABLE 2

\begin{tabular}{|c|c|c|c|}
\hline$(c \stackrel{k}{=} 1)^{1}$ & $Z, Z^{\prime}$ & $(\gamma, \delta)$ & $h\left(Z, Z^{\prime}\right)^{2}$ \\
\hline $\begin{array}{r}2 \\
3 \\
6 \\
7 \\
71 \\
14\end{array}$ & 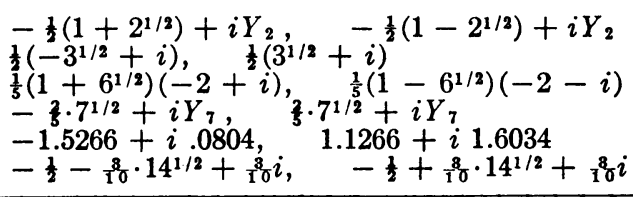 & $\begin{array}{l}(1, \omega) \\
(1,0) \\
(1,0) \\
(3+\omega, 2+\omega) \\
(2+\omega,-2) \\
(4+\omega, 11+3 \omega)\end{array}$ & $\begin{array}{l}.475 \cdots \\
.25 \\
.20 \\
.245 \cdots \\
.129 \cdots \\
.09\end{array}$ \\
\hline$(c=2)^{2}$ & \multirow[b]{2}{*}{ 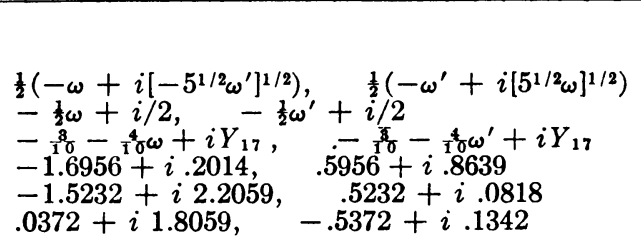 } & \multirow[b]{2}{*}{$\begin{array}{l}(1,0) \\
(2, \omega) \\
(2-\omega,-\omega) \\
(\omega, 2+\omega) \\
(2,1+\omega) \\
(1,0)\end{array}$} & \multirow[b]{2}{*}{$\begin{array}{l}.559 \cdots \\
.25 \\
.405 \cdots \\
.174 \cdots \\
.180 \cdots \\
.242 \cdots\end{array}$} \\
\hline $\begin{array}{r}5 \\
13 \\
17 \\
21 \\
29 \\
33\end{array}$ & & & \\
\hline
\end{tabular}

Possible Low-Points

${ }^{1}$ Recall $\omega=k^{1 / 2}$ and $\omega^{\prime}=-k^{1 / 2}$ for $c=1$ and $\omega=\left(1+k^{1 / 2}\right) / 2, \omega^{\prime}=\left(1-k^{1 / 2}\right) / 2$ for $c=2$.

(5. $\left.417^{1 / 2}-21\right) / 200$. 
output values of $\gamma$ and $\delta$ are listed in Table 2 for the reader's convenience in reconstructing the optimum, (but naturally, several distinct $(\gamma, \delta)$ may be equally capable of producing $\|\gamma Z+\delta\|=1$ ).

7. Bounding Surfaces. We now return to the question of how complicated the floor of the fundamental domain $\boldsymbol{R}$ really is. In every sense, we are establishing a minimum level of complexity, since our grid may not be fine enough to catch all hypersurfaces which make up the floor.

Let us look at the floor, however, in terms of zones. Let us define $\boldsymbol{Z}_{\gamma, \delta}$ (for $\gamma \neq 0)$ the $(\gamma, \delta)$-zone as the set of $\left(Z, Z^{\prime}\right)$ in $U \times U$ for which

$$
\|\gamma Z+\delta\| \leqq\|\gamma(Z+\rho)+\delta\|
$$

for every $\rho \in O$. Then the portion of the floor lying on $\|\gamma Z+\delta\|=1$ can be translated (by $\rho$ ) so as to lie on $\boldsymbol{Z}_{\gamma, \delta}$. In a sense, then, the simplest possible description of the floor would consist of the enumeration of the values of the residue classes $\delta(\bmod \gamma)$. The translations $($ by $\rho)$ involve an additional consideration of complexity which we thereby ignore.

We list the values of $\delta(\bmod \gamma)$ in Table 3 omitting the case $\gamma=1(\delta=0)$, which always occurs (since the point $Z=i, Z^{\prime}=i$ must necessarily lie on the floor

TABLE 3

Analytic Segments of the Floor: $|\gamma Z+\delta|^{2}\left|\gamma^{\prime} Z^{\prime}+\delta^{\prime}\right|^{2}=1$

\begin{tabular}{|c|c|c|c|}
\hline \multirow{2}{*}{$(c \stackrel{k}{=} 1)$} & Input & \multicolumn{2}{|l|}{ Output } \\
\hline & $\gamma$ (where $\left.|N(\gamma)|<2 k / c^{2}\right)$ & $\delta(\bmod \gamma)(\operatorname{excl} . \gamma=1)$ & $\max _{|N(\gamma)|}$ \\
\hline $\begin{array}{l}2 \\
3 \\
6\end{array}$ & $\begin{array}{l}1,2^{1 / 2} \\
1,1+3^{1 / 2}, 3^{1 / 2}, 2 \\
1,2+6^{1 / 2}, 3+6^{1 / 2}, 2,1 \pm 6^{1 / 2}, 6^{1 / 2}\end{array}$ & $\begin{array}{l}1\left(\bmod 2^{1 / 2}\right) \\
1\left(\bmod 1+3^{1 / 2}\right) \\
1\left(\bmod 2+6^{1 / 2}\right) ; \pm 1\left(\bmod 3+6^{1 / 2}\right)\end{array}$ & $\begin{array}{l}2 \\
2 \\
4\end{array}$ \\
\hline 7 & $\begin{array}{c}4+2 \cdot 6^{1 / 2}, 3,4 \pm 6^{1 / 2} \\
1,3+7^{1 / 2}, 2 \pm 7^{1 / 2}, 2,1 \pm 7^{1 / 2}, 7^{1 / 2} \\
6+2 \cdot 7^{1 / 2}, 3,4 \pm 7^{1 / 2}, 4 \pm 2 \cdot 7^{1 / 2}\end{array}$ & $\begin{array}{c}1+6^{1 / 2}(\bmod 2) \\
1\left(\bmod 3+7^{1 / 2}\right) ; \pm 1\left(\bmod 2 \pm 7^{1 / 2}\right)\end{array}$ & 4 \\
\hline 11 & $\begin{array}{l}1,3+11^{1 / 2}, 2,4 \pm 11^{1 / 2}, 2 \pm 11^{1 / 2} \\
6+2 \cdot 11^{1 / 2}, 3,11^{1 / 2}, 4,9+3 \cdot 11^{1 / 2}\end{array}$ & $\begin{array}{l}1\left(\bmod 3+11^{1 / 2}\right) ; 1,14^{1 / 2}(\bmod 2) ; \\
\quad \pm 1, \pm 2\left(\bmod 4 \pm 11^{1 / 2}\right) ; \pm 2, \pm 3\end{array}$ & 7 \\
\hline 14 & $\begin{array}{l}1,4+14^{1 / 2}, 2,3 \pm 14^{1 / 2}, 7+2 \cdot 14^{1 / 2} \\
\quad 8+2 \cdot 14^{1 / 2}, 3,2 \pm 14^{1 / 2}, 5 \pm 14^{1 / 2} \\
1 \pm 14^{1 / 2}, 14^{1 / 2}, 4,12+3 \cdot 14^{1 / 2} \\
6 \pm 2 \cdot 14^{1 / 2}\end{array}$ & $\begin{array}{l}1\left(\bmod 4+14^{1 / 2}\right) ; 1,1+14^{1 / 2}(\bmod \\
2) ; \pm 2\left(\bmod 3 \pm 14^{1 / 2}\right) ; \pm 3(\bmod \\
\left.2 \pm 14^{1 / 2}\right)\end{array}$ & 10 \\
\hline \multicolumn{4}{|l|}{$(c=2)$} \\
\hline 5 & & & 1 \\
\hline 13 & $1, \frac{1}{2}\left[1 \pm 13^{1 / 2}\right], 2$ & $\begin{array}{l}\left.\frac{1}{2}\left[1 \pm 13^{1 / 2}\right](\bmod 2) ; \pm 1\left(13^{1 / 2}\right]\right) \\
\frac{1}{3}[1 \pm 1(\bmod \\
\end{array}$ & 3 \\
\hline 17 & $\begin{array}{l}1, \frac{1}{2}\left[3 \pm 17^{1 / 2}\right], 2, \frac{1}{2}\left[1 \pm 17^{1 / 2}\right], 3 \pm \\
17^{1 / 2}, \frac{1}{2}\left[7^{1} \pm 17^{1 / 2}\right]\end{array}$ & $1\left(\bmod \frac{1}{2}\left[3 \pm 17^{1 / 2}\right]\right)$ & 2 \\
\hline 21 & $\begin{array}{l}1, \frac{1}{2}\left[3+21^{1 / 2}\right], 2, \frac{1}{2}\left[1 \pm 21^{1 / 2}\right] \\
\frac{1}{2}\left[7+21^{1 / 2}\right], 3\end{array}$ & $\begin{array}{c} \pm 1\left(\bmod \frac{1}{2}\left[3+21^{1 / 2}\right]\right) ; \frac{1}{2}\left[1 \pm 21^{1 / 2}\right] \\
(\bmod 2) ; \pm 2\left(\bmod \frac{1}{3}\left[1 \pm 21^{1 / 2}\right]\right)\end{array}$ & 5 \\
\hline 29 & $\begin{array}{l}1,2, \frac{1}{2}\left[3 \pm 29^{1 / 2}\right], \frac{1}{2}\left[1 \pm 29^{1 / 2}\right], 3 \\
\frac{1}{2}\left[9^{1 / 2} \pm 29^{1 / 2}\right]\end{array}$ & $1, \frac{1}{2}\left[1 \pm 29^{1 / 2}\right](\bmod 2) ; \pm 2(\bmod$ & 5 \\
\hline 33 & $\begin{array}{l}1, \frac{1}{2}\left[5 \pm 33^{1 / 2}\right], 6+33^{1 / 2}, \frac{1}{2}\left[7 \pm 33^{1 / 2}\right] \\
2,1_{2}\left[3 \pm 33^{1 / 2}\right], \frac{1}{2}\left[1 \pm 33^{1 / 2}\right], 5 \pm \\
33^{1 / 2}, 3,11+2 \cdot 33^{1 / 2}, 12 \pm 2 \cdot 33^{1 / 2} \\
\frac{1}{2}\left[9 \pm 33^{1 / 2}\right], 4,7 \pm 33^{1 / 2} \\
\quad \frac{1}{2}\left[41 \pm 7 \cdot 33^{1 / 2}\right]\end{array}$ & $1\left(\bmod \frac{1}{2}\left[5 \pm 33^{1 / 2}\right]\right)$ & 2 \\
\hline
\end{tabular}


only on hypersurface $\|Z\|=1)$. In several cases the values of $\delta(\bmod \gamma)$ were also drawn from supplementary runs which were made to explore the neighborhoods of the low-points in Table 2.

We do take symmetries $\left(X \leftrightarrow-X, X^{\prime} \leftrightarrow-X^{\prime}\right)$ and $\left(Z \leftrightarrow Z^{\prime}\right)$ into account, so that for every $\delta(\bmod \gamma)$ occurring, $-\delta(\bmod \gamma)$ and $\pm \delta^{\prime}\left(\bmod \gamma^{\prime}\right)$ are also present; they are listed separately insofar as $\delta \not \equiv-\delta(\bmod \gamma)$ and $\gamma$ and $\gamma^{\prime}$ are nonassociated, etc.

There are 12 "pieces" present (and translates) e.g., for $k=14$ (i.e., 12 residue classes $\delta \bmod \gamma$ including $\gamma=1$ ). There is no reason to be sure that any listing is complete but we might feel relatively sure of the smaller $k$. At any rate, an actual count of output pairs $(\gamma, \delta)$ for $k=5$ yields 7 different pairs $(\gamma, \delta)$ for the simple floor; namely, $\gamma=1$ and $\delta=0, \pm 1, \frac{1}{2}\left( \pm 1 \pm 5^{1 / 2}\right)$. By contrast, an actual count of output values for $k=14$ yields 51 pairs $(\gamma, \delta)$ belonging to the 12 "pieces" (and 39 translates).

8. Concluding Remarks. (a) In calculating $\delta$ and $\delta^{\prime}$ from (3.9), the procedure used is to note that if $\delta=d_{1}+d_{2} \omega$ and $\delta^{\prime}=d_{1}+d_{2} \omega^{\prime}$ then $d_{1}=\psi_{2}\left(\delta, \delta^{\prime}\right)$ and $d_{2}=\psi_{2}\left(\delta, \delta^{\prime}\right)$ by just solving linear equations. Hence, if $\delta_{1}<\delta<\delta_{2}$ and $\delta_{1}^{\prime}<\delta^{\prime}<$ $\delta_{2}{ }^{\prime}$, it is clear that

$$
\psi_{1}\left(\delta_{1}, \delta_{1}{ }^{\prime}\right)<d_{1}<\psi_{1}\left(\delta_{2}, \delta_{2}{ }^{\prime}\right), \quad \psi_{2}\left(\delta_{1}, \delta_{2}{ }^{\prime}\right)<d_{2}<\psi_{2}\left(\delta_{2}, \delta_{1}{ }^{\prime}\right) .
$$

Thus to explore the range (3.9) it is only necessary to explore the range (8.1) for integers $d_{1}$ and $d_{2}$.

(b) To avoid losing a value of $d_{1}$ or $d_{2}$ by round-off errors, the computer artificially rounds up (or down) by actually using not (8.1), but

$$
-.01+\psi_{1}\left(\delta_{1}, \delta_{1}{ }^{\prime}\right) \leqq d_{1} \leqq+.01+\psi_{1}\left(\delta_{2}, \delta_{2}{ }^{\prime}\right) \text {, etc. }
$$

Likewise, equation (3.12) is interpreted as $2 / c^{2} k^{2} \leqq S_{2} \leqq 1.1$, partly to make allowance for the cases $\left(R_{1}=0, R_{2}=0\right)$ where $S_{2}=1$ theoretically.

(c) We are constantly being reminded of our dependence on the "decimal world". By an undeserved stroke of luck, the conjectured low point for $k=6$ (see Table 2) appears as the print-out

$$
R_{1}=-.4, \quad R_{2}=-.4, \quad S_{1}=-1.0, \quad S_{2}=.2005 \text { (calc.). }
$$

Here $R_{1}, R_{2}, S_{1}$ falls in our scanning range (3.13) since $\Delta R_{1}, \Delta R_{2}$, and $\Delta S_{1}$ were taken as .1 for lack of any more inspired choice. Likewise the counterexample (in $\S 5$ ) for the nonsimplicity proof for $k=3$ is a fortunate result of "good decimal" choices for $\Delta R_{1}, \Delta R_{2}$, and $\Delta S_{1}$.

(d) In the counterexamples of $\S 5$ for $k=2$ (or $k=13$ ) the printouts suggest that $Z=-\frac{1}{2} \omega+i Y$ and $Z=-\frac{1}{2} \omega^{\prime}+i Y^{\prime}$ will do for a range or values of $Y$ and $Y^{\prime}$ not too far from the ones attempted; e.g., $Y=1$ and $Y^{\prime}=\frac{1}{2}$ (or $\frac{1}{4}$ as long) as $Y Y^{\prime}=$ $\frac{1}{2}$ (or $\frac{1}{4}$ ). The printouts do not suggest a reason for any freedom for $Y$ and $Y^{\prime}$ in the choice of counterexamples.

The actual computing time was roughly 250 points/minute and this includes, on the average, 6 bisections for the location of $S_{2}$. The running time seemed largely independent of the number of input values $\gamma$ (which varied from 1 when $k=5$ to 
23 when $k=33)$. This may be partly due to the fact that the high internal speed makes the output (to tape) a lengthly process causing some stability (in the ratio of 250 points/minute). Meanwhile, for many input values of $\gamma$, the inequalities (3.9) provide no value of $\delta$ to test. The time for the total computation was roughly one hour spread over several weeks in July and September, 1964.

University of Arizona

Tucson, Arizona

1. H. CoHn, A Second Course in Number Theory, Wiley, New York, 1962.

2. H. CoHs, "On the shape of the fundamental domain of the Hilbert modular group," Theory of Numbers, A. L. Whiteman (Ed.), Proc. Sympos. Pure Math. Vol. 8, Amer. Math. Soc., Providence, R. I., 1965, pp. 190-202.

3. R. H. DeVore, The Lowest Points in the Fundamental Domain of the Hilbert Modular Group for $Q\left(5^{1 / 2}\right)$ and $Q\left(2^{1 / 2}\right)$, Ph.D. Dissertation, University of Arizona, Tucson, Ariz., 1964.

4. J. NymanN, Ideal Structure in Relative-quadratic Fields arising from Fixed-points of the Hilbert Modular Group, Ph.D. Dissertation, University of Arizona, Tucson, Ariz., 1965.

5. C. L. Sieger, Lectures on Advanced Analytic Number Theory, Tata Institute, 1961. 